\title{
THE CHINESE CITRUS FLY, Bactrocera minax (DIPTERA: TEPHRITIDAE): A REVIEW OF ITS BIOLOGY, BEHAVIOUR AND AREA-WIDE MANAGEMENT
}

\author{
M. A. RASHID ${ }^{1}$, Y. DONG ${ }^{1}$, A. A. ANDONGMA ${ }^{1}$, Z. CHEN ${ }^{1}$, \\ Y. WANG ${ }^{1}$, P. XU ${ }^{1}$, P. $\mathrm{LI}^{2}$, P. YANG ${ }^{2}$, A. R. CLARKE ${ }^{3}$ AND \\ C. NIU $^{1}$
}

${ }^{1}$ Hubei Key Laboratory of Insect Resource Application and Sustainable Pest Control, College of Plant Science \& Technology, Huazhong Agricultural University,

Wuhan 430070, China; niuchangying88@163.com

${ }^{2}$ Pest Control Division, National Agricultural Technology Extension and Service

Center, Ministry of Agriculture, Beijing 100125, China

${ }^{3}$ School of Earth, Environmental and Biological Sciences, Faculty of Science and Technology, Queensland University of Technology (QUT), P.O. Box 2434, Brisbane QLD 4001, Australia

\begin{abstract}
SUMMARY
The Chinese citrus fly Bactrocera minax (Enderlein) is a major pest of citrus in some Asian countries. It is a univoltine, oligophagous pest, which strictly infests Citrus species and varieties, and has an exceptionally long pupal diapause. B. minax has great socio-economic importance in China and its neighbouring countries because citrus production is a key fruit industry in these countries. We review the biology and management of this pest with a focus on its distribution, life cycle, diapause, behavioural ecology, and host preferences. We further review potential area-wide integrated pest management (AW-IPM) strategies, including chemical control, but also various eco-friendly, locally developed and adopted techniques applied mainly in China and Bhutan. After years of continuous efforts in AW-IPM of B. minax, significant progress has been achieved in suppressing $B$. minax populations to a level of less than $5 \%$ infested fruit and a $60-80 \%$ reduction in the use of synthetic insecticides against this pest in China.
\end{abstract}

Key Words: temperate fruit fly, life cycle, behavioural ecology, diapause, Tetradacus, Dacinae, oranges, Citrus, AW-IPM, China, Bhutan, IPM

J. Hendrichs, R. Pereira and M. J. B. Vreysen (eds.), Area-Wide Integrated Pest Management: Development and Field Application, pp. 143-159. CRC Press, Boca Raton, Florida, USA. (C) 2021 IAEA 


\section{INTRODUCTION}

Citrus fruits rank first across the world in the international fruit trade in terms of value (Liu et al. 2012; Srivastava 2012). The Chinese citrus fly Bactrocera minax (Enderlein) (Diptera: Tephritidae) is a major pest of Citrus spp. in China, Bhutan, India, Viet Nam and other neighbouring countries (White and Wang 1992; Dong et al. 2014a). It is a univoltine insect with a long pupal diapause that feeds solely on different Citrus species and varieties (Chen et al. 2016). The Chinese citrus fly is thought to be endemic to China as its presence could have been recorded in a poem written about 1000 years ago during the Song dynasty:

"The yellow oranges drop to the ground of the garden due to the wind in autumn. When the oranges opened, there were maggots inside the oranges instead of dragon" (Yang et al. 2013).

In the 1940s B. minax was only recorded in Guizhou and Sichuan, China (Chen and Wong 1943). Currently, it is reported to occur in the major citrus growing provinces of China (Chongqing, Guangxi, Guizhou, Hubei, Hunan, Shanxi, and Sichuan, see Fig. 1), climatically ranging from temperate to subtropical (Wang and Zhang 2009; Gao et al. 2013).

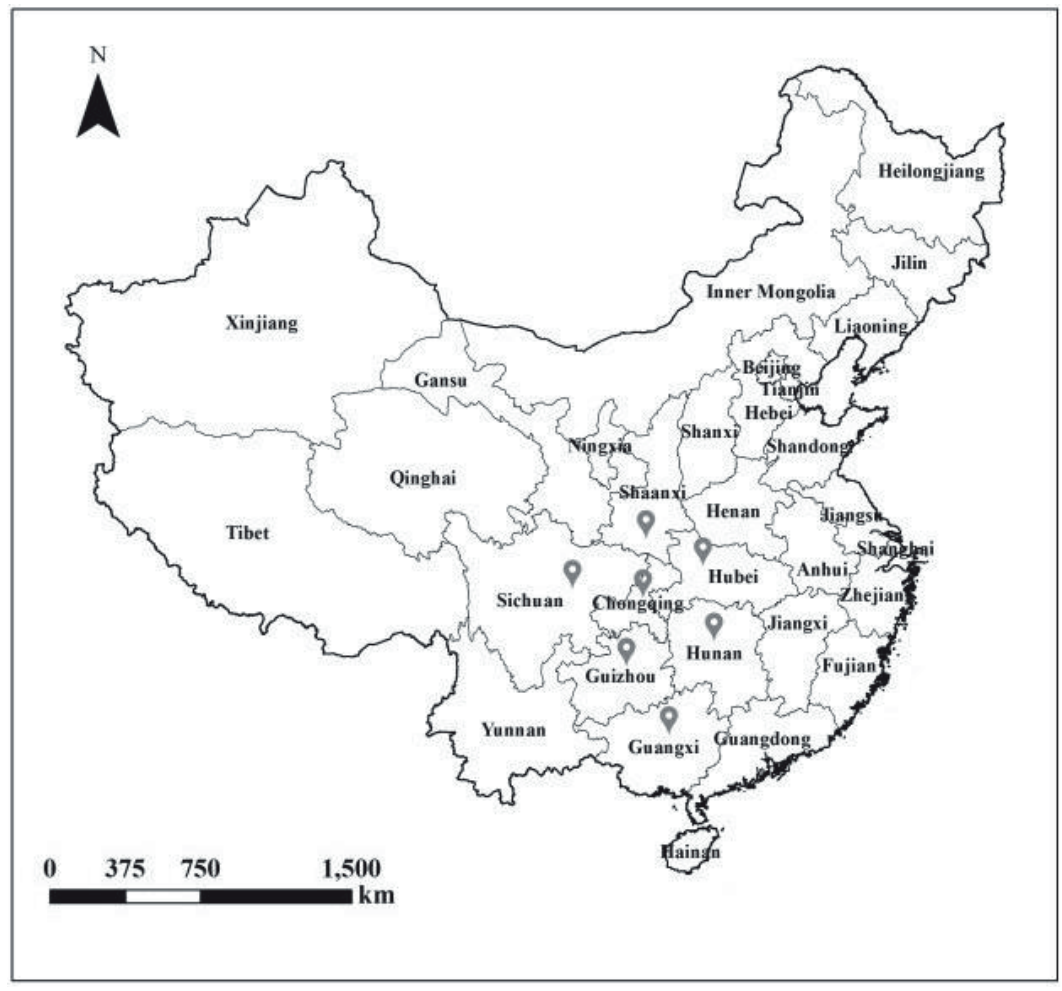

Figure 1. The occurrence and distribution of Bactrocera minax in China (indicated with dark marks showing location within different provinces). 
Following a population outbreak in 2008 in south-western China, and the resulting heavy losses incurred by farmers, $B$. minax was removed from the national quarantine plant pest list in 2009 (Announcement No. 1216 of the Ministry of Agriculture of the People's Republic of China). Thus, the current management against $B$. minax in China has shifted from eradication to suppression.

\subsection{Nature of Damage and Economic Importance}

The adult female fly oviposits its eggs under the peel of green, immature citrus fruits with the aid of its elongated ovipositor (Fig. 2). The eggs hatch when fruit reach midlevel development and the larvae feed on the fruit flesh leading to premature ripening and fruit drop, ultimately resulting in economic damage and yield loss (Allwood et al. 1999; Liu et al. 2015). The larval stage is therefore considered as the most destructive life stage (Dorji et al. 2006). Heavy economic losses of USD 200 million were reported in 2008 due to the above-mentioned outbreak of B. minax in Guangyuan, Sichuan Province, China. Almost 1 million tons of oranges were destroyed during this flare-up. This outbreak and heavy infestation resulted in a ban on international trade of citrus commodities from China. The outbreak was reported by "The China Daily" in its headlines to highlight the serious damage to the citrus industry (Liu et al. 2015).

Similarly, B. minax is a major pest in the eastern Himalayan kingdom of Bhutan, where mandarin Citrus reticulata Blanco is one of the major fruit crops. Crop losses caused by $B$. minax infestation ranging from 35 to $75 \%$ are common in mid- and highaltitude orchards $(>1100 \mathrm{~m})$, and the fly is considered as one of the major barriers to citrus production (van Schoubroeck 1999; Dorji et al. 2006; Xia et al. 2018).

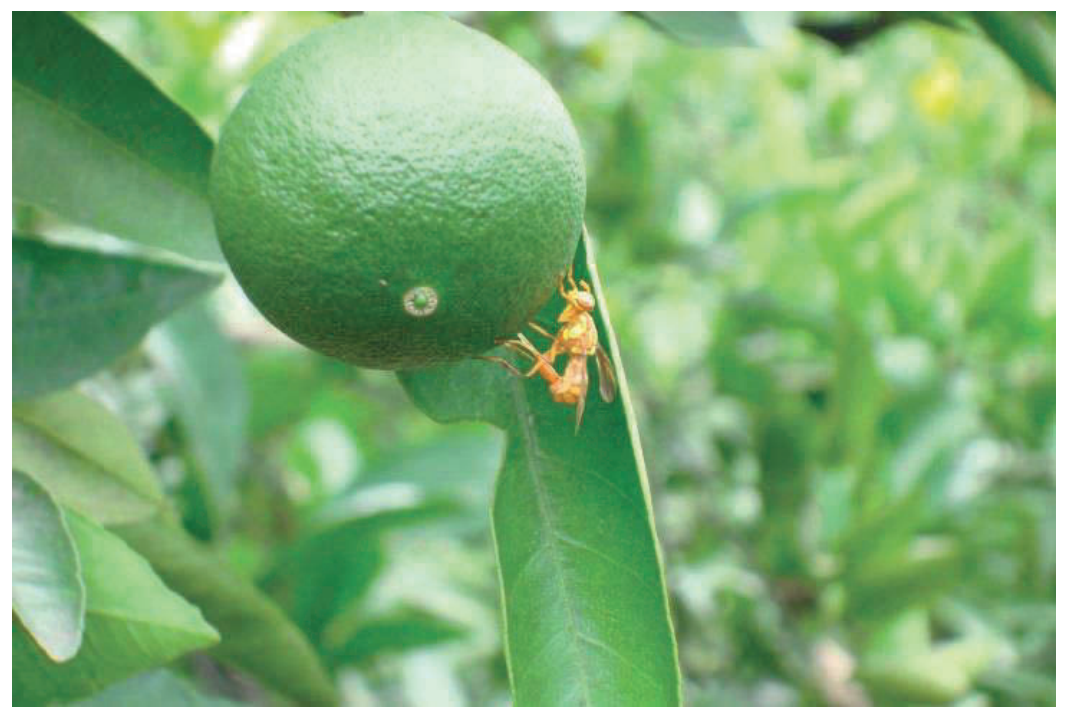

Figure 2. Female Bactrocera minax using its elongated ovipositor to lay its eggs into small green citrus fruit (20 30 mm diameter). 


\subsection{Taxonomy, Distribution and Host Plants}

The Chinese citrus fly was for the first time described in 1920 from specimens collected from Sikkim, India by Enderlein and named Polistomimetes minax (Thompson 1998). In 1940, the species was also collected in Sichuan Province, China. Drew (1979) provided a detailed description of the B. minax based on the specimens collected in 1920 and placing the species in the subgenus Bactrocera (Polistomimetes).

Subsequently, a lectotype of $B$. minax was ascribed to the subgenus Bactrocera (Tetradacus) by White and Wang (1992), who also recorded that Bactrocera citri Chen should be regarded as a junior synonym of $B$. minax. The fly is currently placed in the subgenus Bactrocera (Tetradacus), a small monophyletic clade evolutionary basal to all other Bactrocera species (Krosch et al. 2012).

Currently, B. minax is regarded as present in Bhutan, China, India (West Bengal and Sikkim), Nepal, and Viet Nam (Dorji et al. 2006; Drew et al. 2006). The host range of Chinese citrus fly is almost exclusively restricted to Citrus species and varieties. It has been recorded in citron Citrus medica L., lemon Citrus limon (L.) Burman f., meiwa kumquat Fortunella crassifolia Swingle, pummelo Citrus maxima (Burm.) Merr., sour orange Citrus aurantium L., sweet orange Citrus sinensis (L.) Osbeck, tangerine Citrus reticulata Blanco, navel orange Citrus sinensis Osb. var. brasiliensis Tanaka, pyunkyul Citrus tangerina Hort. ex Tanaka, grapefruit Citrus paradisi Macfad., and trifoliate orange Poncirus trifoliata L. (Nath 1972; Chao and Ming 1986; Liu et al. 2014). Among these, the preferred citrus host plant is sweet orange (Liu et al. 2014).

\section{BIOLOGY AND LIFE CYCLE}

\subsection{Seasonal Phenology}

The phenology of $B$. minax's life cycle may vary subtly depending on local climate conditions. However, the general pattern appears quite fixed. Based on the population of $B$. minax in Guizhou, China and in Bhutan, we summarize the life cycle of $B$. minax as follows:

1. The female oviposits eggs in small unripe fruit from mid-June to mid-July. Usually it takes two months for the eggs to hatch, much longer than other species in the Bactrocera genus; the eggs hatch in late August, after which the larvae go through three larval instars.

2. The larval stage lasts until the end of October, which is then followed by a pupal stage.

3. To survive the cold winter temperatures, the pupae enter a six-month overwintering diapause.

4. Adult emergence usually begins in May and mating starts about 25 to 30 days after adult emergence (Wang and Luo 1995; Dorji et al. 2006). 


\subsection{Eggs of B. minax}

The egg of $B$. minax is milky white, oblong and curved in shape, slightly pointed at one end and round at the other. It has a length of 1.1 to $1.5 \mathrm{~mm}$ and a maximum width of 0.2 to $0.4 \mathrm{~mm}$ (Sun 1961). Female B. minax lay eggs in clutches. Usually, each female oviposits about 14 to 17 eggs per oviposition event, with a maximum of 35 eggs per clutch. During its lifetime, a female can produce more than 100 eggs (Zhang 1989). After depositing eggs, the fruit surface is covered with juice around the oviposition wound. In the beginning, this juice is transparent to translucent for one to three days, then it gradually becomes yellow. The oviposition site bulges out, cracks and fruit skin around the oviposition site turns yellow to crimson-purple (Wang and Zhang 1993). Egg hatch starts in July and reaches its peak in late August (Xiong et al. 2016).

\subsection{Larvae of B. minax}

After eclosion, the larva feeds internally on the citrus flesh. The mature larva is milky white or pale yellow, 15 to $18 \mathrm{~mm}$ long, conical in shape and nearly transparent at one end. The mouth is equipped with sclerotized mouth hooks, and the body has 11 segments. The larval stage lasts 52 to 72 days depending on temperature, with an average of 63 days (Lu et al. 1997). Young larvae usually feed in a small group on a single fruit segment, later spreading to other segments: the average number of larvae in an infected fruit is 9.5 (Zhang 1989). Larval infestation leads to premature fruitfall from October to November (Liu et al. 2015).

The larvae stay within the fruit for about 18-52 days after fruit drop. Such a long pre-pupal period is very unusual among the Dacinae and it suggests that long larval development occurs after fruit drop (Dorji et al. 2006). A mature, third instar larva usually leaves the fruit in the early morning and pupates within a day. Pupation starts in late October and reaches a peak in early to mid-November (Dong et al. 2013; Chen et al. 2016).

\subsection{Pupae of B. minax}

The mature third instar larva pupates in the soil at 3 to $5 \mathrm{~cm}$ depth (Zhang 1989; Dorji et al. 2006). The long overwintering pupal diapause in B. minax is highly unusual within the genus Bactrocera and is considered as an adaptive strategy to survive the cold winter periods which occur in its native range (Fan et al. 1994). In Yichang city, where $B$. minax is present, the mean winter temperature usually ranges from 5 to 15 ${ }^{\circ} \mathrm{C}$ (Dong et al. 2013). The overwintering pupal phase lasts for 160-170 days, with the emergence of adults synchronised with the early fruiting season of citrus (Wang and Luo 1995). The pupa of $B$. minax is 9 to $10 \mathrm{~mm}$ long with a diameter of $4 \mathrm{~mm}$, weight average of $77.5 \mathrm{mg}$. It is oval in shape and yellow-brown in colour. Prior to adult emergence, the pupal case becomes slightly dark brown (Zhang 1989; Wang and Luo 1995). 


\subsection{Diapause Termination in B. minax}

Research on the development of efficient and sustainable $B$. minax management techniques is still very difficult because the fly has only one generation per year, accompanied by six months of pupal diapause (White and Wang 1992; van Schoubroeck 1999). This bottleneck made the mass-production of this fly very difficult, thereby limiting research capability and its potential use in area-wide integrated pest management (AW-IPM) programmes that have a Sterile Insect Technique (SIT) component (Lü et al. 2014). Therefore, options to break the diapause are considered essential for research and the development of the SIT package for this fly (see Section 4.2.3).

Pupal diapause is usually an evolved response in univoltine temperate tephritids in order to survive harsh environmental conditions and seasonal periods of host scarcity (Teixeira and Polavarapu 2001, 2005; Ragland et al. 2009; Papanastasiou et al. 2011; Moraiti et al. 2012). In B. minax pupal diapause is a vital strategy to tolerate cold stress and face the seasonal adversity. Research into the underlying mechanisms in terms of diapause termination, such as major cellular shifts, protein processing, differentially expressed genes and pathways, are still ongoing (Lü et al. 2014; Dong et al. 2014a; Wang et al. 2016, 2017).

Pupal diapause in B. minax is influenced by both chilling temperature and duration. A higher chilling temperature, coupled with longer chilling duration, results in a shorter pupal developmental time and improves the synchronisation of adult emergence (Dong et al. 2013). Apart from providing this chilling exposure, hormonal application of 20-hydroxyecdysone (20E) is considered a faster and more efficient method to break pupal diapause in B. minax (Dong et al. 2014a; Wang et al. 2014; Chen et al. 2016). Either injection or topical application of $20 \mathrm{E}$ can trigger a rapid termination of the pupal diapause in $B$. minax and the morphological changes are observed within 1 week at $22^{\circ} \mathrm{C}$. On the tenth day after $20 \mathrm{E}$ treatment, the head, thorax and abdomen of the insect can clearly be distinguished, and the colour of body and eyes are milky (Chen et al. 2016). The 20E early-response genes, including ecr, broad and foxo, are up-regulated within $72 \mathrm{~h}$ of $20 \mathrm{E}$ exposure, indicating these genes are involved in diapause termination processes and pupal metamorphosis (Chen et al. 2016).

The gene sets involved in protein and energy metabolisms vary throughout earlylate- and post-diapause insects in response to cold stress. When diapause is terminated by $20 \mathrm{E}$, many genes involved in ribosome and metabolic pathways are differentially expressed, which may mediate diapause transition (Dong et al. 2014a). The variation of transcriptomic and metabolomic profiles of pupae at five stages (pre, early-, middle-, late-, and post-diapause) suggests major shifts in metabolism and signal transduction, as well as changes in the endocrine and digestive systems. Nine metabolites significantly contribute to the variation in the metabolomic profiles, especially proline and trehalose, which are well-known cryoprotective agents (Wang et al. 2017). 


\subsection{Adults of B. minax}

The adult fly is 10 to $13.2 \mathrm{~mm}$ long, not including the female ovipositor, with a wingspan of $\sim 10.8 \mathrm{~mm}$ (Drew 1979). The female possesses a long ovipositor of approximately $6.5 \mathrm{~mm}$. The flies are of a brownish colour with yellow markings, the wings have a dark band along the outer margin, and the general appearance is wasplike (Chen and Xie 1955). A morphological description of the adult is provided in Drew et al. (2007), who also note that the fly is probably the largest of all Bactrocera species (Fig. 2).

\section{BEHAVIOUR AND ECOLOGY}

Since the Chinese citrus fly is not attracted to methyl eugenol or cue-lure as are many species of the genus Bactrocera, thorough behavioural and ecological studies have been carried out over the past years in order to develop effective monitoring and control strategies in the long run.

\subsection{Feeding Behaviour}

It is well known that for most studied tephritid species, both males and females are anautogenous and forage for sugar and protein to fuel metabolic activities and to meet reproductive requirements (Aluja and Norrbom 1999; Drew and Yuval 2000; Taylor et al. 2013). Females need a protein diet for vitellogenesis and ovarian development (Harwood et al. 2015), while males feed on protein to reach sexual maturity leading to copulation which is crucial in achieving reproduction (Lushchak et al. 2013). Adult $B$. minax forage on non-host plants for honeydew, nectar, sooty mould and fruit juices to meet their dietary requirements during sexual maturation. The flies then shift to licking sooty moulds, bird faeces and, to a lesser extent, an unknown substance (probably leaf phylloplane bacteria and plant leachates) on citrus leaves and fruits during the mating and oviposition period (Hendrichs et al. 1993; Dong et al. 2014b).

\subsection{Mating Behaviour}

Male aggression and territoriality have been reported as a typical behaviour in some Bactrocera species (Shelly 1999; Weldon 2005; Benelli et al. 2014, 2015). For most tropical polyphagous Bactrocera species, males aggregate at a common place on foliage to attract and court females for mating, i.e. a non-resource-based lek mating system (Emlen and Oring 1977; Maan and Seehausen 2011). However, in B. minax, as in many temperate and oligophagous tephritids, male courtship behaviour is absent and the mating system is a resource-based defence polygyny, consisting of two phases: 1) males defend a resource (host fruit) (intrasexual selection), 2) where copulation takes place (intersexual selection) (Opp et al. 1996). In the wild, all mating events take place on citrus fruit (Dong et al. 2014b). Territory formation and copulation usually occurs on immature green fruits. It has been suggested that male flies that try to copulate in the vicinity of the ovipositional site have more chances to encounter and court receptive females (Prokopy 1976; Smith and Prokopy 1980). 
Mating behaviour in $B$. minax is closely synchronised with the host fruiting season and has been described in the field as follows: (i) the male establishes its territory close to a potential oviposition substrate (citrus fruit); (ii) the female lands on the fruit and begins inspection and ovipositor probing on the fruit surface; and (iii), the male mounts and copulates with the female (Dong et al. 2014b). In this mating system females face trade-offs associated with the cost of additional, apparently unneeded matings on each fruit in return for access to resources. Like in the case of Rhagoletis species the resource is assumed to be the oviposition site (Opp et al. 1996; Opp and Prokopy 2000; Prokopy and Papaj 2000).

\subsection{Oviposition and Host Preference}

In tephritids, fruit flies use different cues for host finding behaviour and egg-laying behaviour. Usually, long-distance volatile chemicals are important before landing on host trees, visual stimuli act as short range once on the tree and contact chemicals on and inside host fruit influence female egg-laying decision.

Bactrocera minax oviposits solely into citrus fruits (Family Rutaceae) (Wang and Luo 1995; Dong et al. 2013). It is a large, powerful insect with a long ovipositor adapted for piercing through the thick skin of young, green citrus fruit (Liu and Zhou 2016). Visual cues including fruit shape, colour, and size are important for host finding (Prokopy and Owens 1983; Piñero et al. 2017). On the other hand, the egglaying behaviour is greatly influenced by chemical stimuli, for example, semiochemicals, sugar content, levels of secondary plant compounds and physical properties of fruit (Bush 1969). The preference of B. minax oviposition on different citrus varieties is as follows, Citrus sinensis cv. Navel and C. aurantium $>C$. sinensis cv. Bintang, Amakusa and C. reticulata cv. Satsuma $>C$. maxima cv. Shatian $>C$. reticulata $\mathrm{cv}$. Ponka. This ovipositional preference is positively correlated with larval survival and development; while in the field greater egg-laying occurs on those citrus fruits which are close to the surrounding vegetation and trees (Liu et al. 2014).

\subsubsection{Visual Cues (Colour, Shape, and Size) for Oviposition}

The hardness of a citrus fruit peel has an impact on the female insect's decision to oviposit (Lin et al. 2011). On the basis of egg oviposition marks on citrus fruit, it appears that $B$. minax significantly prefers to oviposit on the distal hemisphere rather than the basal hemisphere (Liu and Zhou 2016). Apart from peel hardness, the ovipositional behaviour of tephritid female flies is also influenced by fruit colour and shape (Alyokhin et al. 2000). It has been shown that tephritid flies respond to fruitmimics of the same colour or reflecting similar levels of light than host fruit of a particular fly species (Aluja and Norrbom 1999). For example, the Queensland fruit fly Bactrocera tryoni (Froggatt) showed attraction to spheres painted with cobalt blue pigments, which reflected the same UV spectrum as favoured blue-coloured host fruits occuring in its native rainforest environment (Drew et al. 2003). The oriental fruit fly Bactrocera dorsalis (Hendel) is attracted to white-yellow colour (Vargas et al. 1991) and the apple maggot Rhagoletis pomonella (Walsh), a pest of apples, is attracted to fruit-mimicking traps such as a red sphere (Duan and Prokopy 1995). 
Both sexes of $B$. minax are attracted to orange or yellow/green spheres of $50 \mathrm{~mm}$ diameter (Drew et al. 2006). However, we found that B. minax adults prefer green over other colours, and this preference is significantly increased in sexually mature flies over immature flies (author submitted results).

\subsubsection{Chemical Cues (Semiochemicals) for Oviposition}

Chemical cues play an important role in foraging and oviposition of fruit flies (Sarles et al. 2015), and these chemicals are widely exploited in integrated pest management (Shrivastava et al. 2010). For example, the application of oviposition marking pheromone reduced Rhagoletis cerasi L. infestation up to $100 \%$ in cherry orchards (Katsoyannos and Boller 1976; Boller and Hurther 1998). Together with visual cues, semiochemical cues may also influence host finding and egg-laying of $B$. minax. The peel odours of different varieties of orange preferred by $B$. minax produce different volatile blends, including acids, aldehydes, alcohols, and oils. It is presumed that these volatile compounds directly influence the olfactory orientation of B. minax females. However, there is as yet no proof if these volatiles released by host plants have a direct impact on the oviposition preference of B. minax (Liu and Zhou 2016).

From the perspective of the biology, ecology and behaviour, B. minax is more reminiscent of flies in the temperate genus Rhagoletis than other pest species in the Bactrocera genus. Therefore, some components of pest management can be drawn from the extensive scientific literature on apple maggot fly $R$. pomonella, European cherry fruit fly Rhagoletis cerasi, and other fruit flies (Vargas et al. 2016). For example, the application of fruit volatiles in conjunction with visual traps may yield good results for the control of B. minax.

\section{TOWARDS THE AREA-WIDE MANAGEMENT OF B. MINAX}

Several different control tactics have been used to manage populations of the Chinese citrus fly. These include chemical control and "attract and kill" techniques using protein/food baits and fruit-mimicking traps. In addition, pilot trials of the Sterile Insect Technique (SIT) have been assessed for B. minax control (Wang et al. 1990; Wang and Luo 1995), and farmers in China and Bhutan have adopted locally developed suppression techniques.

\subsection{Use of Chemicals}

Pesticide applications (cover sprays) are the most commonly used conventional control practices against insects pests, especially in the case of outbreaks. Though they are effective in reducing the losses caused by fruit fly infestation, the negative impacts of pesticides on humans, the environment and non-target organisms have raised much concern. Different insecticides have been used to suppress $B$. minax populations, including phoxim, dichlorvos, chlorpyrifos, abamectin, botanically derived pesticides, and pyrethroids. Amongst these, abamectin and dichlorvos proved to have the highest and lowest toxicity, respectively. However, chlorpyrifos had the strongest effect on pupae, and phoxim had the strongest influence on emergence. 
These chemicals are not recommended due to toxicity to non-target organisms and long residual effects, but they are effective against $B$. $\operatorname{minax}$ (Liu et al. 2015).

\subsection{Eco-friendly Management}

\subsubsection{Field Sanitation}

Field sanitation is an effective and important strategy to reduce $B$. minax populations for the next fruiting season. The collection of infested fruits from the ground every week from mid-September to late-November is essential to remove the breeding population from orchards. The protocol demands that these infested fruits are transferred into thick plastic bags (20-25 kg per bag), that can be supplemented with aluminium phosphide to facilitate the killing of larvae. However, this is not critical if the bags are kept in the field under the sun for 7 10 days. Finally, the rotting fruits serve as fertilizer. The plastic bags can be recycled and used again (Liu et al. 2011; Li et al. 2013).

In Bhutan, cultural practices such as the application of soil tillage, along with natural predation (pupae picking by birds), seem to have a role in reducing the number of pupae. However, this reduction is not significant. Thus, it is not recommended as the only control measure for reducing the overall $B$. minax population in the wild (Dorji et al. 2010).

\subsubsection{Protein and Food Baits}

Spraying a mixture of protein bait, with a small quantity of insecticide added, has proven to be an effective strategy for large-scale control of fruit fly populations (Conway and Forrester 2011). Bait sprays are effective for fruit fly population control as newly emerged females require protein to become sexually mature (Perez-Staples et al. 2007; McQuate 2009). In China, it is a widely accepted approach for the farmers to use vinegar, sugar and wine mixtures, plus detergent, as baits station/spots spray for the control of B. minax, which is simple and cheap (Zhou et al. 2012). Attractants such as GF-120, and other locally available commercial products, are also used for the suppression of the Chinese citrus fly. Fresh enzymatically-hydrolysed beer yeast (H-protein) liquid protein bait effectively attracted and killed more B. minax flies than GF-120 sprayed in the field (Zhou et al. 2012).

\subsubsection{Use of Fruit-Mimicking Traps}

Usually semiochemicals and plant derived volatiles are used to trap fruit flies (DíazFleischer et al. 2014), but the males of $B$. minax are not attracted to either of the standard Bactrocera male lures (i.e. methyl eugenol and cue-lure) (Drew et al. 2006). Visual traps have been used as an alternative, and in Bhutan, both sexes of the fly were most attracted to green-yellow or orange fruit-mimicking spheres in the field (Drew et al. 2006). In recent years, a specific fruit-mimicking trap (spherical green sticky trap) has been developed and widely applied to monitor and control B. minax in China. After field deployment of spherical traps in sweet orange orchards in Zigui, China, the infested fruit rate dropped to $2.7 \%$ compared with $28.6 \%$ in untreated control orchards (Yi et al. 2015). Efficiency of the control effort was closely 
associated with appropriate trap deployment density and time (Chen et al. 2017). Considering the cost of commercial traps, as well as efficacy, spherical green sticky traps with a diameter of $7 \mathrm{~cm}$ were recommended at a deployment rate of 20 30 traps per $1000 \mathrm{~m}^{2}$ in citrus orchards (Chen et al. 2017; Gong et al. 2017).

\subsubsection{Sterile Insect Technique (SIT)}

The SIT has been successfully used to manage fruit flies including Ceratitis capitata (Wiederman), B. dorsalis, the melon fly Zeugodacus cucurbitae (Coquillett) and the Mexican fruit fly Anastrepha ludens (Loew) (Calkins et al. 1994; Koyama et al. 2004; Dhillo et al. 2005; Enkerlin et al. 2017). In AW-IPM programmes that have an SIT component, the production of sterile insects in large numbers is of paramount importance (Enkerlin 2021). Sterilisation can be achieved by irradiation or genetic manipulation. Irradiating $B$. minax pupa two days before emergence with a dose of $90 \mathrm{~Gy}$ is recommended to ensure adult sterility (Zhang and Li 1990).

Due to the serious damage in major citrus production regions in China, a massrelease of sterilized B. minax flies was carried out in Guizhou, China in the late 1980s and early 1990s. In these pilot projects, mature $3^{\text {rd }}$ instar larvae were collected in fruit from the field and then allowed to pupate in the lab (Wang et al. 1990; Wang and Luo 1995). Although the SIT trials in Guizhou Province resulted in a significant reduction of the pest population (Wang et al. 1990; Wang and Luo 1995), the technology is so far not integrated into B. minax control programmes in China. This is mainly related to problems with the development of mass-rearing methods due to technical barriers such as the extreme long pupal diapause period. This is the reason that there is currently no foreseeable plan for an SIT approach against B. minax, although the selection of non-diapausing strains is being explored.

\subsubsection{Natural Enemies}

Knowledge on the parasitoids associated with the Chinese citrus fly remains very scarce. Only one parasitoid, Diachasmimorpha feijeni van Achterberg, has been associated with this fly in Bhutan (van Achterberg 1999) and in China (authors' unpublished data). However, there is still no detailed information available on the parasitoid's interactions with Chinese citrus fly or the potential for parasitoid manipulation.

\subsubsection{From Pilot Trials to Area-Wide Management in China}

Since 2009, the Ministry of Agriculture and National Agro-Tech Extension and Service Center of China has promoted and organized annual nation-wide conferences and training courses on tephritid control for local technicians and farmers, with the aim to educate and transfer new developments and technologies. Over the years, pilot trials of AW-IPM approaches against $B$. minax have been implemented in the provinces of Guizhou, Hubei, Hunan, Shanxi, and Sichuan where B. minax is a serious pest. The validated demonstration practices in the main citrus producing regions led to the establishment of a $B$. minax AW-IPM programme in China. 
In the beginning of every year, the Ministry of Agriculture announces and issues online management programmes for $B$. minax control, along with that for other major agricultural pests, to facilitate the sustainable management of the pests. In the case of $B$. minax, the integrated environmental-friendly measures can be summarized as follows:

- First, population monitoring in April using field traps and pupal emergence cages, aimed at accurately identifying the timing of pupal developmental state and adult emergence, is carried out by local plant protection stations annually: this is critical to guide the implementation of control practices.

- Second, "attract and kill" strategies are applied from May to July including the systematic use of spherical green sticky traps, protein bait sprays and sugarvinegar-wine liquid in bait stations or spot sprays.

- Third, field sanitation of habitats/orchards by removing the fallen and infested fruits and weeds from September to November has proved to be an important and effective population control measure. Recyclable plastic bags are widely adopted to keep-and-kill the mature larvae by a combination of hypoxia and heat.

Thus, through years of continuous efforts in AW-IPM of $B$. minax, significant progress has been achieved in suppressing $B$. minax populations to a level resulting in less than $5 \%$ of infested commercial fruit and a reduction in the use of synthetic pesticides by $60-80 \%$. The $B$. minax AW-IPM programme will now be complying with national standards for 'green control', which results in notable direct and indirect economic, ecological and social benefits for China.

\section{FUTURE PERSPECTIVES}

With increased understanding of the biology and behaviour of B. minax, effective operational AW-IPM strategies against this pest have been established in China and Bhutan. However, SIT and natural enemies have not yet been exploited due to various biological and physiological obstacles. Great efforts are required to overcome these gaps for the future sustainable management of $B$. minax:

1. Mass-rearing of $B$. minax still remains a big challenge considering its univoltine and oligophagous traits. Future work should be focused on improving (a) understanding of egg hatch and (b) artificial diet formulation for the newly hatched young larvae.

2. Little information has been published on the insect-plant interactions of $B$. minax. It is widely known that the adults have a close relationship with Citrus spp., but how these adults utilize visual, olfactory and tactile cues to orientate to host plants for mating and oviposition has received very little attention.

3. Symbiotic organisms, including Wolbachia (Stouthamer et al. 1999), that affect biology and reproduction of $B$. minax should be characterized. A thorough screening of microorganisms by culture-dependent and high-throughput technology, in combination with related functional studies, will help to better understand the complex relationship between symbionts and $B$. minax. Such knowledge may lead to potential development and application of the Incompatible Insect Technique (IIT) (Zabalou et al. 2004). 
4. Natural enemies have not been used as a component of AW-IPM against $B$. minax most likely because very little knowledge is available about them and their effectiveness. The possible synchronised diapause of parasitoids and $B$. minax pupae needs to be investigated. In addition, other agents such as predators or fungi causing pupal mortality deserve to be further investigated.

5. There are currently no effective semiochemical or plant derived volatile lures to attract $B$. minax males or females available. The volatile chemicals from the host fruits, as well as a sex attractant for monitoring and mass-trapping the Chinese citrus fly, urgently needs to be identified and exploited.

6. The growing published online resources on transcriptome, proteome, and genome of $B$. minax, RNA interference and CRISPR-Cas9 technologies targeting specific gene functions, will facilitate further investigations of molecular mechanisms responsible for the biology, behaviour, physiology and evolution of the Chinese citrus fly. The comprehensive understanding of $B$. minax is the most promising way to develop sustainable management of this economically important citrus pest in the long run.

\section{ACKNOWLEDGEMENTS}

The authors thank all the reviewers who enthusiastically made comments and suggestions to improve this paper. This study was funded by National Natural Science Foundation of China (31661143045, 31371945), International Atomic Energy Agency (CRP No. 17153 and No. 18269), Crop Disease and Insect Pest Monitoring and Control Program supported by the Ministry of Agriculture of People's Republic of China (10162130108235049) and the Fundamental Research Funds for the Central Universities (2662015PY148).

\section{REFERENCES}

Allwood, A., A. Chinajariyawong, S. Kritsaneepaiboon, R. Drew, E. Hamacek, D. Hancock, C. Hengsawad, J. Jipanin, M. Jirasurat, and C. K. Krong. 1999. Host plant records for fruit flies (Diptera: Tephritidae) in Southeast Asia. Raffles Bulletin of Zoology 47: 1-92.

Aluja, M., and A. Norrbom. 1999. Fruit flies (Tephritidae): Phylogeny and evolution of behavior. CRC Press, Boca Raton, Florida, USA. 984 pp.

Alyokhin, A. V., R. H. Messing, and J. J. Duan. 2000. Visual and olfactory stimuli and fruit maturity affect trap captures of Oriental fruit flies (Diptera: Tephritidae). Journal of Economic Entomology 93: 644-649.

Benelli, G., K. M. Daane, A. Canale, C. Y. Niu, R. H. Messing, and R. I. Vargas. 2014. Sexual communication and related behaviours in Tephritidae: Current knowledge and potential applications for Integrated Pest Management. Journal of Pest Science 87: 385-405.

Benelli, G., N. Desneux, D. Romano, G. Conte, R. H. Messing, and A. Canale. 2015. Contest experience enhances aggressive behaviour in a fly: When losers learn to win. Scientific Reports 5: 9347.

Boller E.F., and J. Hurther. 1998. The marking pheromone of the cherry fruit fly: A novel non-toxic and ecologically safe technique to protect cherries against cherry fruit fly infestation, pp 99-101. In Proceedings International Symposium on Insect Pheromones. March 1998. Wageningen, The Netherlands.

Bush, G. L. 1969. Sympatric host race formation and speciation in frugivorous flies of genus Rhagoletis (Diptera: Tephritidae). Evolution 23: 237-251. 
Calkins, C. O., K. Bloem, S. Bloem, and D. L. Chambers. 1994. Advances in measuring quality and assuring good field performance in mass reared fruit flies, pp. 85-96. In C. O. Calkins, W. Klassen, and P. Liedo (eds.), Fruit flies and the Sterile Insect Technique. CRC Press, Boca Raton, Florida, USA.

Chao, Y., and Y. Ming. 1986. The investigation on fruit-flies (Trypetidae-Diptera) injurious to fruits and vegetables in south China. Technical Bulletin of Plant Quarantine Research 10: 1-61.

Chen, F., and F. Wong. 1943. Study on citrus maggot in Jiangjin County. Agricultural Science 1: 46.

Chen, S. X., and Y. Z. Xie. 1955. The classification and characterization of Bactrocera (Tetradacus) minax Enderlein. Acta Entomologica Sinica 5: 123.

Chen, Z. Z., Y. C. Dong, Y. H. Wang, A. A. Andongma, M. A. Rashid, P. Krutmuang, and C. Y. Niu. 2016. Pupal diapause termination in Bactrocera minax: An insight on 20-hydroxyecdysone induced phenotypic and genotypic expressions. Scientific Reports 6: 27440.

Chen, Z. Z., A. S. Deng, Q. Zhu, W. B. Chen, X. M. Xu, H. B. Zheng, and C. Y. Niu. 2017. Assessing attracting and killing effects of the spherical traps against Chinese citrus fly, Bactrocera minax. Journal of Huazhong Agricultural University 36: 33-37.

Conway, H. E., and O. T. Forrester. 2011. Efficacy of ground spray application of bait sprays with malathion or spinosad on Mexican fruit fly (Diptera: Tephritidae) in Texas citrus. Journal of Economic Entomology 104: 452-458.

Dhillo, M. K., R. Singh, J. S. Naresh, and H. C. Sharma. 2005. The melon fruit fly, Bactrocera cucurbitae: A review of its biology and management. Journal of Insect Science 5: 1-16.

Díaz-Fleischer, F., J. C. Piñero, and T. E. Shelly. 2014. Interactions between tephritid fruit fly physiological state and stimuli from baits and traps: Looking for the pied piper of Hamelin to lure pestiferous fruit flies, pp. 145-172. In T. E. Shelly, N. Epsky, E. B. Jang, J. Reyes-Flores, and R. Vargas (eds.), Trapping and the detection, control, and regulation of tephritid fruit flies. Springer, Dordrecht, The Netherlands.

Dong, Y. C., N. Desneux, C. L. Lei, and C. Y. Niu. 2014a. Transcriptome characterization analysis of Bactrocera minax and new insights into its pupal diapause development with gene expression analysis. International Journal of Biological Sciences 10: 1051-1063.

Dong, Y. C., L. Wan, R. Pereira, N. Desneux, and C. Y. Niu. 2014b. Feeding and mating behaviour of Chinese citrus fly Bactrocera minax (Diptera, Tephritidae) in the field. Journal of Pest Science 87: 647-657.

Dong, Y. C., Z. J. Wang, A. R. Clarke, R. Pereira, N. Desneux, and C. Y. Niu. 2013. Pupal diapause development and termination is driven by low temperature chilling in Bactrocera minax. Journal of Pest Science 86: 429-436.

Dorji, C., A. R. Clarke, R. A. Drew, B. S. Fletcher, P. Loday, K. Mahat, S. Raghu, and M. C. Romig. 2006. Seasonal phenology of Bactrocera minax (Diptera: Tephritidae) in western Bhutan. Bulletin of Entomological Research 96: 531-538.

Dorji, C., K. Mahat, and P. Loday. 2010. Effect of tillage on pupal mortality of the Chinese citrus fruit fly, Bactrocera minax (Enderlein) (Diptera:Tephritidae). Journal of Renewable Natural Resources 6: 24-30.

Drew, R. 1979. The genus Dacus Fabricius (Diptera: Tephritidae)-two new species from northern Australia and a discussion of some subgenera. Australian Journal of Entomology 18: 71-80.

Drew, R. A. I., and B. Yuval. 2000. The evolution of fruit fly feeding behavior, pp. 731-749. In M. Aluja and A. Norrbom (eds.), Fruit flies (Tephritidae): Phylogeny and evolution of behavior. CRC Press, Boca Raton, Florida, USA.

Drew, R. A. I., R. J. Prokopy, and M. C. Romig. 2003. Attraction of fruit flies of the genus Bactrocera to colored mimics of host fruit. Entomologia Experimentalis et Applicata 107: 39-45.

Drew R. A. I., M. C. Romig, and C. Dorji. 2007. Records of dacine fruit flies and new species of Dacus (Diptera: Tephritidae) in Bhutan. The Raffles Bulletin of Zoology 55: 1-21.

Drew, R. A. I., C. Dorji, M. C. Romig, and P. Loday. 2006. Attractiveness of various combinations of colors and shapes to females and males of Bactrocera minax (Diptera: Tephritidae) in a commercial mandarin grove in Bhutan. Journal of Economic Entomology 99: 1651-1656.

Duan, J. J., and R. J. Prokopy. 1995. Control of apple maggot flies (Diptera: Tephritidae) with pesticidetreated red spheres. Journal of Economic Entomology 88: 700-707.

Emlen, S. T., and L. W. Oring. 1977. Ecology, sexual selection, and the evolution of mating systems. Science 197: 215-223. 
Enkerlin, W. R. 2021. Impact of fruit fly control programmes using the Sterile Insect Technique, pp. $977-$ 1004. In V. A. Dyck, J. Hendrichs and A. Robinson (eds.), Sterile Insect Technique - Principles and practice in Area-Wide Integrated Pest Management. Second Edition. CRC Press, Boca Raton, Florida, USA.

Enkerlin W. R., J. M. Gutiérrez Ruelas, R. Pantaleón, C. Soto-Litera, A. Villaseñor-Cortés, J.L. Zavala-López, D. Orozco-Dávila, P. Montoya-Gerardo, L. Silva-Villarreal, E. Cotoc-Roldán, F. Hernández-López, A. Arenas-Castillo, D. Castellanos-Domínguez, A. Valle-Mora, P. RendónArana, C. Cáceres-Barrios, D. Midgarden, C. Villatoro-Villatoro, E. Lira-Prera, O. ZelayaEstradé, R. Castañeda-Aldana, J. López-Culajay, P. Liedo-Fernández, G. Ortíz-Moreno, J. Reyes-Flores, F. Ramírez y Ramírez, J. Trujillo-Arriaga, J. Hendrichs. 2017. The Moscamed regional programme: A success story of area-wide Sterile Insect Technique application. Entomologia Experimentalis et Applicata 264(3): 188-203.

Fan, J. A., X. Q. Zhao, and J. Zhu. 1994. A study on the cold-resistance and diapause in Tetradacus citri Chen. Journal of Southwest Agricultural University 6: 532-534.

Gao, L. Z., Y. H. Liu, X. W. Wan, J. Wang, and F. Hong. 2013. Screening of microsatellite markers in Bactrocera minax (Diptera: Tephritidae). Scientia Agricultura Sinica 46: 3285-3292.

Gong, B. Y., F. L. Xiao, B. C. Mo, W. G. Dong, Z. Q. Wen, S. Z. Yang, and X. X. Li. 2017. Research on the attracting effects of the spherical traps against Chinese citrus fly, Bactrocera minax. Plant Protection 43: 218-221.

Harwood, J. F., K. Chen, P. Liedo, H. G. Müller, J. L. Wang, A. E. Morice, and J. R. Carey. 2015. Female access and diet affect insemination success, senescence and the cost of reproduction in the male Mexican fruit fly Anastrepha ludens. Physiological Entomology 40(1): 65-71.

Hendrichs, J., S. S.Cooley, and R. J. Prokopy. 1993. Uptake of plant surface leachates by apple maggot flies, pp. 173-175. In M. Aluja, and P. Liedo (eds.), Fruit flies, biology and management. Springer, New York, NY, USA.

Katsoyannos B. I., and E. F. Boller. 1976. First field application of oviposition-deterring marking pheromone of European cherry fruit fly. Environmental Entomology 5:151-152.

Koyama, J., H. Kakinohana, and T. Miyatake. 2004. Eradication of the melon fly, Bactrocera cucurbitae, in Japan: Importance of behavior, ecology, genetics, and evolution. Annual Review of Entomology 49: 331-349.

Krosch, M. N., M. K. Schutze, K. F. Armstrong, G. C. Graham, D. K. Yeates, and A. R. Clarke. 2012. A molecular phylogeny for the tribe Dacini (Diptera: Tephritidae): Systematic and biogeographic implications. Molecular Phylogenetics \& Evolution 64: 513-523.

Li, P., C. Y. Niu, Q. He, G. Z. Zhou, X. M. Xu, P. Y. Yang, H. B. Peng, Z. C. Jiang, M. Zhang, F. Ren, Y. H. Wang, W. Z. Hu, and J. X. Yang. 2013. A poster on green control strategies for Bactrocera minax. Agriculture Press, Beijing, China.

Lin, W. L., S. Z. Yang, M. S. Pan, H. L. Chen, Z. P. Huang, J. G. Long, and F. L. Xiao. 2011. The damage characteristics of Tetradacus citri Chen on different Citrus varieties in Hunan Province. Hunan Agricultural Sciences 23: 95-97.

Liu, C., X. H. Xiao, H. H. Wu, C. H. Yang, and C. J. Song. 2011. Experimental trial on plastic bag use to manage the Chinese citrus fruit fly larvae infested fruits. Plant Doctor 24: 32-34.

Liu, H. Q., G. F. Jiang, Y. F. Zhang, F. Chen, X. J. Li, J. S. Yue, C. Ran, and Z. M. Zhao. 2015. Effect of six insecticides on three populations of Bactrocera (Tetradacus) minax (Diptera: Tephritidae). Current Pharmaceutical Biotechnology 16: 77-83.

Liu, L., and Q. Zhou. 2016. Olfactory response of female Bactrocera minax to chemical components of the preference host citrus volatile oils. Journal of Asia-Pacific Entomology 19: 637-642.

Liu, L., Q. Zhou, A. Q. Song, and K. X. You. 2014. Adult oviposition and larval feeding preference for different Citrus varieties in Bactrocera minax (Diptera: Tephritidae). Acta Entomologica Sinica 57: $1037-1044$.

Liu, Y. Q., E. Heying, and S. A. Tanumihardjo. 2012. History, global distribution, and nutritional importance of citrus fruits. Comprehensive Reviews in Food Science and Food Safety 11: 530-545.

Lu, H. X., K. P. He, H. F. Ruan, and B. Z. Mou. 1997. The biological features of Chinese citrus fly Dacus citri (Chen). Journal of Hubei Agricultural College 17: 169-170.

Lü, Z., L. Wang, G. Zhang, F. Wan, J. Guo, H. Yu, and J. Wang. 2014. Three heat shock protein genes from Bactrocera (Tetradacus) minax Enderlein: Gene cloning, characterization, and association with diapause. Neotropical Entomology 43: 362-372. 
Lushchak, V., D. V. Gospodaryov, B. M. Rovenko, I. S. Yurkevych, N. V. Perkhulyn, and V. I. Lushchak. 2013. Specific dietary carbohydrates differentially influence the life span and fecundity of Drosophila melanogaster. The Journals of Gerontology Series A: Biological Sciences and Medical Sciences 69: 3-12.

Maan, M. E., and O. Seehausen. 2011. Ecology, sexual selection and speciation. Ecology Letters 14: 591602.

McQuate, G. 2009. Effectiveness of GF-120NF fruit fly bait as a suppression tool for Bactrocera latifrons (Diptera: Tephritidae). Journal of Applied Entomology 133: 444-448.

Moraiti, C. A., C. T. Nakas, and N. T. Papadopoulos. 2012. Prolonged pupal dormancy is associated with significant fitness cost for adults of Rhagoletis cerasi (Diptera: Tephritidae). Journal of Insect Physiology 58: 1128-1135.

Nath, D. 1972. Callantra minax (Enderlein) (Tephritidae: Diptera), a new record of a ceratitinid fruit fly on orange fruits (Citrus reticulata Blanco) in India. Indian Journal of Entomology 34: 246.

Opp, S. B., S. A. Spisak, A. Telang, and S. S. Hammond. 1996. Comparative mating systems of two Rhagoletis species: The adaptive significance of mate guarding, pp. 43-50. In B. A. McPheron, and G. J. Steck (eds.), Fruit fly pests: A world assessment of their biology and management. St. Lucie Press, Delray Beach, Florida, USA.

Opp, S. B., and R. J. Prokopy. 2000. Multiple mating and reproductive success of male and female apple maggot flies, Rhagoletis pomonella (Diptera: Tephritidae). Journal of Insect Behavior 13: 901-914.

Papanastasiou, S. A., D. Nestel, A. D. Diamantidis, C. T. Nakas, and N. T. Papadopoulos. 2011. Physiological and biological patterns of a highland and a coastal population of the European cherry fruit fly during diapause. Journal of Insect Physiology 57: 83-93.

Perez-Staples, D., V. Prabhu, and P. W. Taylor. 2007. Post-teneral protein feeding enhances sexual performance of Queensland fruit flies. Physiological Entomology 32: 225-232.

Piñero, J.C., S. K. Souder, R. I. Vargas. 2017. Vision-mediated exploitation of a novel host plant by a tephritid fruit fly. PLoS One 12(4): e0174636.

Prokopy, R. J. 1976. Feeding, mating, and oviposition activities of Rhagoletis fausta flies in nature. Annals of the Entomological Society of America 69: 899-904.

Prokopy, R. J., and D. R. Papaj. 2000. Behavior of flies of the genera Rhagoletis, Zonosemata, and Carpomya (Trypetinae: Carpomyina), pp. 219-252. In M. Aluja, and A. L. Norrbom (eds.), Fruit flies (Tephritidae): Phylogeny and evolution of behavior. CRC Press, Boca Raton, Florida, USA.

Prokopy R. J., and E. D. Owens. 1983. Visual detection of plants by herbivorous insects. Annual Review of Entomology 28: 337-364.

Ragland, G. J., J. Fuller, J. L. Feder, and D. A. Hahn. 2009. Biphasic metabolic rate trajectory of pupal diapause termination and post-diapause development in a tephritid fly. Journal of Insect Physiology 55(4): 344-350.

Sarles, L., A. Verhaeghe, F. Francis, and F. J. Verheggen. 2015. Semiochemicals of Rhagoletis fruit flies: Potential for Integrated Pest Management. Crop Protection 78: 114-118.

Shelly, T. E. 1999. Defense of oviposition sites by female Oriental fruit flies (Diptera: Tephritidae). Florida Entomologist 82: 339-346.

Shrivastava, G., M. Rogers, A. Wszelaki, D. R. Panthee, and F. Chen. 2010. Plant volatiles-based insect pest management in organic farming. Critical Reviews in Plant Sciences 29: 123-133.

Smith, D. C., and R. J. Prokopy. 1980. Mating behavior of Rhagoletis pomonella (Diptera: Tephritidae) VI. Site of early-season encounters. The Canadian Entomologist 112(6): 585-590.

Srivastava, A. K. 2012. Advances in citrus nutrition. Springer, Dordrecht, The Netherlands. 477 pp.

Stouthamer, R., J. Breeuwer, and G. Hurst. 1999. Wolbachia pipientis: Microbial manipulator of arthropod reproduction. Annual Reviews in Microbiology 53: 71-102.

Sun, Z. Y. 1961. The study and control of Dacus citri. Chinese Plant Protection Science, Scientific Publishing House, Beijing, China.

Taylor, P., D. Pérez-Staples, C. Weldon, S. Collins, B. Fanson, S. Yap, and C. Smallridge. 2013. Postteneral nutrition as an influence on reproductive development, sexual performance and longevity of Queensland fruit flies. Journal of Applied Entomology 137: 113-125.

Teixeira, L. A., and S. Polavarapu. 2001. Postdiapause development and prediction of emergence of female blueberry maggot (Diptera: Tephritidae). Environmental Entomology 30: 925-931.

Teixeira, L. A., and S. Polavarapu. 2005. Diapause development in the blueberry maggot Rhagoletis mendax (Diptera: Tephritidae). Environmental Entomology 34: 47-53. 
Thompson, F. C. 1998. Fruit fly expert identification system and systematic information database: A resource for identification and information on fruit flies and maggots, with information on their classification, distribution and documentation. Backhuys Publishers for the North American Dipterists' Society, Leiden, Netherlands.

van Achterberg, C. 1999. The Palaearctic species of the genus Diachasmimorpha Viereck (Hymenoptera: Braconidae: Opiinae). Zoologische Mededeelingen 73: 1-10.

van Schoubroeck, F. 1999. Learning to fight a fly: Developing citrus IPM in Bhutan. Unpublished PhD thesis, Wageningen Universiteit, The Netherlands.

Vargas, R., J. D. Stark, R. J. Prokopy, and T. A. Green. 1991. Response of Oriental fruit fly (Diptera: Tephritidae) and associated parasitoids (Hymenoptera: Braconidae) to different-color spheres. Journal of Economic Entomology 84: 1503-1507.

Vargas, R. I., J. C. Piñero, L. Leblanc, N. C. Manoukis, and R. F. L. Mau. 2016. Area-wide management of fruit flies (Diptera: Tephritidae) in Hawaii, pp. 673-693. In S. Ekesi, S. Mohamed, and M. Meyer (eds.), Fruit fly research and development in Africa: Towards a sustainable management strategy to improve horticulture. Springer International Publishing, Switzerland.

Wang, H. S., and H. Q. Zhang. 1993. Control of the Chinese citrus fly, Dacus citri (Chen), using the Sterile Insect Technique, pp. 505-512. In Proceeding: Management of insect pests: Nuclear and related molecular and genetic techniques. FAO/IAEA International Symposium, 19-23 October 1992, Vienna, Austria. STI/PUB/909. IAEA, Vienna, Austria.

Wang, H. S., C. D. Zhao, H. X. Li, H. Z. Lou, Q. R. Liu, W. Tang, J. G. Hu, and H. Q. Zhang. 1990. Control of Chinese citrus fly Dacus citri by male sterile technique. Acta Agriculturae Nucleatae Sinica 4(3): 135-138.

Wang, J., H. Fan, K. C. Xiong, and Y. H. Liu. 2017. Transcriptomic and metabolomic profiles of Chinese citrus fly, Bactrocera minax (Diptera: Tephritidae), along with pupal development provide insight into diapause program. PLoS One 12: e0181033.

Wang, J., H. Y. Zhou, Z. M. Zhao, and Y. H. Liu. 2014. Effects of juvenile hormone analogue and ecdysteroid on adult eclosion of the fruit fly Bactrocera minax (Diptera: Tephritidae). Journal of Economic Entomology 107: 1519-1525.

Wang, J., K. C. Xiong, and Y. H. Liu. 2016. De novo transcriptome analysis of Chinese citrus fly, Bactrocera minax (Diptera: Tephritidae), by high-throughput illumina sequencing. PLoS One 11: e0157656.

Wang, X. J., and L. Y. Luo. 1995. Research progress in the Chinese citrus fruit fly. Entomological Knowledge 32: 310-315.

Wang, X. L., and R. J. Zhang. 2009. Review on biology, ecology and control of Bactrocera (Tetradacus) minax Enderlein. Journal of Environmental Entomology 31: 73-79.

Weldon, C. W. 2005. Mass-rearing and sterilisation alter mating behaviour of male Queensland fruit fly, Bactrocera tryoni (Froggatt) (Diptera: Tephritidae). Australian Entomology 44: 158-163.

White, I. M., and X. Wang. 1992. Taxonomic notes on some dacine (Diptera: Tephritidae) fruit flies associated with citrus, olives and cucurbits. Bulletin of Entomological Research 82: 275-279.

Xia, Y., X. L. Ma, B. H. Hou, and G. C. Ouyang. 2018. A review of Bactrocera minax (Diptera: Tephritidae) in China for the purpose of safeguarding. Advances in Entomology 6: 35-61.

Xiong, K. C., J. Wang, J. H. Li, Y. Q. Deng, P. Pu, H. Fan, and Y. H. Liu. 2016. RNA interference of a trehalose-6-phosphate synthase gene reveals its roles during larval-pupal metamorphosis in Bactrocera minax (Diptera: Tephritidae). Journal of Insect Physiology 91: 84-92.

Yang, W. S., C. R. Li, J. Lan, H. L. An. 2013. The spread mode and dispersal history of Chinese citrus fly. Journal of Yangtze University 10: 8-11.

Yi, J. P., S. H. Li, G. G. Zhang, F. Xiang, G. Z. Zhou, and H. G. Luo. 2015. Attracting and killing effects of the green-yellow spherical traps against Chinese citrus fly, Bactrocera minax. China Plant Protection 35: 34-37.

Zabalou, S., A. Apostolaki, I. Livadaras, G. Franz, A. Robinson, C. Savakis, and K. Bourtzis. 2009. Incompatible Insect Technique: Incompatible males from a Ceratitis capitata genetic sexing strain. Entomologia Experimentalis et Applicata 132: 232-240.

Zhang, W., and Y. Y. Li. 1990. Effect of ${ }^{60} \mathrm{Co} \gamma$ irradiation on germ cell of Chinese citrus fly Dacus citri. Acta Agriculturae Nucleatae Sinica 4: 115-119.

Zhang, Y. A. 1989. Citrus fruit flies of Sichuan province (China). EPPO Bulletin 19: 649-654.

Zhou, X. W., C. Y. Niu, P. Han, and N. Desneux. 2012. Field evaluation of attractive lures for the fruit fly Bactrocera minax (Diptera: Tephritidae) and their potential use in spot sprays in Hubei province (China). Journal of Economic Entomology 105: 1277-1284. 